\title{
Synaptic proteins in CSF as potential novel biomarkers for prognosis in prodromal Alzheimer's disease
}

Flora H. Duits ${ }^{1 * \dagger}$, Gunnar Brinkmalm ${ }^{2,3+}$, Charlotte E. Teunissen ${ }^{1,4}$, Ann Brinkmalm²,3, Philip Scheltens ${ }^{1}$, Wiesje M. Van der Flier ${ }^{1,5}$, Henrik Zetterberg ${ }^{2,3,6,7}$ and Kaj Blennow ${ }^{2,3}$

\begin{abstract}
Background: We investigated whether a panel of 12 potential novel biomarkers consisting of proteins involved in synapse functioning and immunity would be able to distinguish patients with Alzheimer's disease (AD) and patients with mild cognitive impairment (MCl) from control subjects.

Methods: We included 40 control subjects, 40 subjects with $\mathrm{MCl}$, and 40 subjects with AD from the Amsterdam Dementia Cohort who were matched for age and sex (age $65 \pm 5$ years, 19 [48\%] women). The mean follow-up of patients with $\mathrm{MCl}$ was 3 years. Two or three tryptic peptides per protein were analyzed in cerebrospinal fluid using parallel reaction monitoring mass spectrometry. Corresponding stable isotope-labeled peptides were added and used as reference peptides. Multilevel generalized estimating equations (GEEs) with peptides clustered per subject and per protein (as within-subject variables) were used to assess differences between diagnostic groups. To assess differential effects of individual proteins, we included the diagnosis $\times$ protein interaction in the model. Separate GEE analyses were performed to assess differences between stable patients and patients with progressive $\mathrm{MCl}(\mathrm{MCl}-\mathrm{AD})$.

Results: There was a main effect for diagnosis $(p<0.01)$ and an interaction between diagnosis and protein $(p<0.01)$. Analysis stratified according to protein showed higher levels in patients with $\mathrm{MCl}$ for most proteins, especially in patients with MCl-AD. Chromogranin A, secretogranin II, neurexin 3, and neuropentraxin 1 showed the largest effect sizes; $\beta$ values ranged from 0.53 to 0.78 for patients with $\mathrm{MCl}$ versus control subjects or patients with $A D$, and from 0.67 to 0.98 for patients with MCl-AD versus patients with stable $\mathrm{MCl}$. In contrast, neurosecretory protein VGF was lower in patients with $A D$ than in patients with $\mathrm{MCl}(B=-0.93$ [SE 0.22]) and control subjects ( $B=0.46$ [SE 0.19]).

Conclusions: Our results suggest that several proteins involved in vesicular transport and synaptic stability are elevated in patients with $\mathrm{MCl}$, especially in patients with $\mathrm{MCl}$ progressing to $\mathrm{AD}$ dementia. This may reflect early events in the AD pathophysiological cascade. These proteins may be valuable as disease stage or prognostic markers in an early symptomatic stage of the disease.
\end{abstract}

Keywords: Parallel reaction monitoring, Synaptic proteins, CSF biomarkers, Alzheimer's disease

\footnotetext{
*Correspondence: f.duits@vumc.nl

${ }^{\dagger}$ Equal contributors

${ }^{1}$ Alzheimer Center and Department of Neurology, Amsterdam Neuroscience,

VU University Medical Center, P.O. Box 7057, 1007MB Amsterdam, The

Netherlands

Full list of author information is available at the end of the article
}

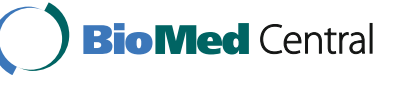

(c) The Author(s). 2018 Open Access This article is distributed under the terms of the Creative Commons Attribution 4.0 International License (http://creativecommons.org/licenses/by/4.0/), which permits unrestricted use, distribution, and reproduction in any medium, provided you give appropriate credit to the original author(s) and the source, provide a link to the Creative Commons license, and indicate if changes were made. The Creative Commons Public Domain Dedication waiver (http://creativecommons.org/publicdomain/zero/1.0/) applies to the data made available in this article, unless otherwise stated. 


\section{Background}

The neuropathological process of Alzheimer's disease (AD) is characterized by accumulation of plaques composed of aggregated amyloid- $\beta$ (A $\beta)$ protein, neurofibrillary tangles consisting of hyperphosphorylated tau, and neuronal degeneration and loss [1]. The cerebrospinal fluid (CSF) biomarkers of these processes-amyloid- $\beta$ 1-42 (A $\left.\beta_{42}\right)$, total tau, and tau phosphorylated at threonine 181 ( $\mathrm{p}$-tau) - show very consistent changes in $\mathrm{AD}$ dementia and prodromal AD [2], and they have been included as evidence for the presence of AD pathology in research diagnostic criteria for $\mathrm{AD}[3,4]$. However, in addition to amyloid and tau pathology, processes such as inflammation and synaptic dysfunction also play an important role and may correlate more directly with cognitive decline [5-7]. Hence, biomarkers for these processes may be valuable for disease monitoring and to predict prognosis or rate of cognitive decline.

On the basis of in-house data derived from unbiased CSF proteomic studies, we selected a panel of synaptic and other proteins for analysis with parallel reaction monitoring (PRM) MS. Explorative proteomics provides a hypothesis-free approach for detecting a large number of proteins and peptides in human body fluids such as plasma and CSF [8]; however, the disadvantage of this technique is the relatively poor reproducibility [9]. PRM is an MS method that focuses on predefined sets of proteins, or peptide peaks from proteolytically digested proteins, thereby allowing higher analytical sensitivity and higher throughput of samples [10,11]. By adding known amounts of isotope-labeled internal standards to the sample, quantification of the actual concentration can be achieved [11]. An advantage over immunoassays is that there is no need for antibodies. Hence, PRM is well suited for validation of explorative proteomic studies [9].

The selection of proteins for the present study was based on a literature review for their possible involvement in AD pathophysiology. The PRM panel consisted of neurosecretory protein VGF (VGF), chromogranin A (CHGA), and secretogranin 2 (SCG2), granins that are presumed to be involved in axonal or synaptic vesicle transport (the granins VGF, CHGA, and SCG2) [12]; cystatin $C(\mathrm{Cys} C)$, a protease involved in $A \beta$ degradation $[13,14] ; \beta_{2}$-microglobulin $\left(\beta_{2} \mathrm{M}\right)$ and lysozyme $C$ (LysC), proteins involved in the innate immune system $[15,16]$; and neurexins (NRXNs) NRXN-1, NRXN-2, and NRXN3 as well as neuronal pentraxin 1 (NPTX1), neurofascin (NFASC), and neurocan core protein (NCANP), proteins involved in synapse formation and stabilization [17-21]. Several of these proteins, including VGF, CHGA, SCG2, $\mathrm{Cys} C$, and $\beta_{2} \mathrm{M}$, have been suggested in previous studies to be involved in AD pathology [12-14, 22-28]. A pilot study that we performed after developing the PRM panel showed promising results, with lower levels for several proteins in patients with $\mathrm{AD}$ dementia compared with control subjects [29]. The aims of the present study were to validate this panel of proteins in a larger and independent cohort, as well as to investigate whether the panel has potential for distinguishing patients with prodromal $\mathrm{AD}$ and patients with $\mathrm{AD}$ dementia from cognitively healthy subjects.

\section{Methods \\ Patients}

All patients were included, based on the availability of CSF, from the memory clinic-based Amsterdam Dementia Cohort [30]. We selected 40 patients who had received a diagnosis of probable AD dementia and matched them for age and sex with 40 patients with subjective cognitive decline (SCD), who served as control subjects, and with 40 patients with mild cognitive impairment (MCI). All subjects underwent extensive cognitive screening at baseline (between 2004 and 2013), including physical and neurological examinations, electroencephalography, magnetic resonance imaging (MRI), and laboratory tests. Neuropsychological investigation included at least one test per cognitive domain, as well as the Mini Mental State Examination (MMSE) for global cognition. Diagnoses were made by consensus of a multidisciplinary team without knowledge of CSF results. AD dementia and MCI were diagnosed according to the criteria of the National Institute of Neurological and Communicative Disorders and Stroke-Alzheimer's Disease and Related Disorders Association criteria and Petersen's criteria, respectively [31, 32], and all patients met the core clinical National Institute on Aging-Alzheimer's Association criteria [3, 33]. When results of all clinical investigations were normal (i.e., criteria for MCI or any psychiatric or neurological disorder not fulfilled), patients were labeled as having SCD. Patients with MCI were included only when there was clinical follow-up of at least 1 year, and the diagnoses of SCD and $\mathrm{AD}$ dementia had to be confirmed at any follow-up visit for subjects to be included in this study. At each follow-up visit, physical, neurological, and neuropsychological examinations were repeated. During follow-up (mean \pm SD $2.8 \pm 1.1$ years), 13 patients with MCI progressed to AD dementia (MCI-AD), 23 patients with $\mathrm{MCI}$ remained stable (sMCI), and 3 patients progressed to another type of dementia. All subjects gave written informed consent for the use of their clinical data and CSF for research purposes. The study was approved by the local ethical review board.

\section{CSF analysis of AD biomarkers}

CSF was obtained by lumbar puncture using a 25-gauge needle and a syringe, and it was collected into $10 \mathrm{ml}$ polypropylene tubes (Sarstedt, Nümbrecht, Germany). 
Part of the CSF was used for routine analysis, including leukocyte and erythrocyte count, glucose concentration, and total protein concentration. Within $2 \mathrm{~h}$, the remaining CSF was centrifuged at $1800 \times g$ for 10 minutes at $4{ }^{\circ} \mathrm{C}$, transferred to new polypropylene tubes, and stored either at $-20{ }^{\circ} \mathrm{C}$ until analysis of $\mathrm{A} \beta_{42}$, tau, and p-tau or directly at $-80{ }^{\circ} \mathrm{C}$ until further analysis. The team involved in CSF analyses was blinded to clinical diagnosis. CSF levels of $A \beta_{42}$, total tau, and p-tau were measured with commercially available enzymelinked immunosorbent assays ( $\beta$ - amyloid $_{(1-42)}$, hTAU$\mathrm{Ag}$, and Phosphotau $(181 \mathrm{P})$; Fujirebio, Ghent, Belgium) on a routine basis as described elsewhere [34]. Measurements took place consecutively within 1 month of the patient's baseline visit. Intra-assay coefficients of variation $(\mathrm{CVs})$ were $($ mean $\pm \mathrm{SD}) 2.0 \pm 0.5 \%$ for $\mathrm{A} \beta_{42}$, $3.2 \pm 1.3 \%$ for tau, and $2.9 \pm 0.8 \%$ for p-tau, and interassay CVs $\left(\right.$ mean $\pm \mathrm{SD}$ ) were $10.9 \pm 1.8 \%$ for $\mathrm{A} \beta_{42}, 9.9 \pm 2.1 \%$ for tau, and $9.1 \pm 1.8 \%$ for p-tau.

\section{APOE genotyping}

For apolipoprotein $\mathrm{E}(A P O E)$ genotyping, DNA was isolated from $10 \mathrm{ml}$ of ethylenediaminetetraacetic acidpreserved blood using the QIAamp DNA blood isolation kit from Qiagen (Hilden, Germany). The genotype was determined with the LightCycler APOE mutation detection kit (Roche Diagnostics GmbH, Mannheim, Germany).

\section{Parallel reaction monitoring MS panel}

The sample preparation and acquisition methods for PRM analysis are described in detail elsewhere [29]. For the present study, we used the same sample preparation and MS methods, with minor modifications as described below. Because quantitative MS analysis of intact proteins is currently not feasible, a number of peptides suitable for MS analysis were obtained by digestion of CSF proteins with trypsin. Using nanoflow LC-MS in a shotgun approach, we injected $1 \mu \mathrm{l}$ of trypsin-digested CSF from patients with and without AD biomarker profiles. Using this approach, we detected more than 2000 tryptic peptides from more than 400 proteins (unpublished data, G. Brinkmalm, Oct 2013). Based on these experiments and the current literature, a number of proteins were selected for further investigation. For each protein, two or three proteotypic peptides were selected. Corresponding stable isotope-labeled peptides were added and used as reference peptides in the PRM analyses. In addition, known amounts of bovine serum albumin (BSA) protein and a corresponding stable isotope-labeled peptide were added to each sample as a reference to monitor sample-processing variations for example digestion. To ensure good-quality data, the mass spectrometer was operated in a relatively slow mode with long injection times (up to 300 milliseconds) per acquisition. Because this limited the maximum number of peptides that could be handled simultaneously, we divided the proteins into two panels. The first panel included CysC, $\beta_{2} \mathrm{M}$, VGF, CHGA, and SCG2, and the second panel included LysC, NRXN-1, NRXN-2, NRXN-3, NPTX1, NFASC, and NCANP. The turnaround time was $72 \mathrm{mi}-$ nutes per sample, which made it impractical to analyze all data on one occasion. Therefore, we divided the dataset into three subsets (randomized according to diagnosis, age, and sex) and performed digestion as well as PRM analysis at different times. This introduced small variations in digestion and LC-MS performance (day-to-day variations). To adjust for these effects, a CSF pool was aliquoted, and four pool samples were used as calibrants in each run of 40 samples. Study samples, CSF pool samples, and heavy isotopelabeled standards were prepared as previously described [29], except that standard 0.5-, 1.5-, and 2.0-ml polypropylene tubes were used in the present work. After thawing, $20 \mu \mathrm{l}$ of the internal standard mixture was added to each sample. Tryptic digestion was performed as previously described [29], except that no shaking of samples was performed. Digested samples were centrifuged, desalted using Oasis 30- $\mu$ m HLB 96well $\mu$ Elution plates (Waters, Milford, MA, USA) with $\mathrm{MeOH}$ for elution into polypropylene plates. Sample eluates were transferred into Eppendorf LoBind vials (Eppendorf, Hauppauge, NY, USA), dried in a SpeedVac (Thermo Fisher Scientific, Waltham, MA, USA), and stored at $-80{ }^{\circ} \mathrm{C}$ pending PRM analysis. Directly prior to PRM analysis, samples were reconstituted in $50 \mathrm{mM} \mathrm{NH} \mathrm{NHCO}_{3}$ and split as desired for each panel. Twenty-five microliters of CSF were used for panel 1 , and $50 \mu \mathrm{l}$ were used for panel 2 .

PRM analysis was performed using a Dionex UltiMate 3000 system (SRD-3600 degasser, WPS-3000TPL autosampler, LPG-3600 pump, FLM-3100 column compartment; Thermo Fisher Scientific) coupled to a Thermo Q Exactive electrospray ionization hybrid quadrupole-Orbitrap high-resolution mass spectrometer (Thermo Fisher Scientific). Separation was performed with a Hypersil GOLD reversed phase column (inner diameter $2.1 \mathrm{~mm}$, length $100 \mathrm{~mm}$; Thermo Fisher Scientific) operated at a flow rate of $100 \mu \mathrm{l} / \mathrm{mi}$ nute. Mobile phase A was $0.1 \%$ formic acid, mobile phase B was $84 \%$ acetonitrile/0.1\% formic acid ( $\mathrm{vol} / \mathrm{vol}$ ), and the gradient length was 50 minutes. CVs of BSA in the CSF pool samples were (mean \pm SD over the three runs) $2.3 \pm 1.1 \%$ in the first PRM panel and $2.3 \pm 1.4 \%$ in the second PRM panel. In the study samples, BSA showed CVs of $2.3 \pm 0.5 \%$ for the first panel and $2.4 \pm 0.1 \%$ for the second panel. Levels correlated highly with each other between the two PRM panels (Pearson's $r=0.92$ ) and did 
not differ between diagnostic groups, but they were slightly different between the subsets subjected to trypsinization and PRM analysis on separate occasions (see Additional file 1). CVs of the proteins of the PRM panel are described in detail elsewhere [29]. The CSF pool samples were used to normalize data between acquisition days. This was done individually for each peptide. Data processing was performed as described elsewhere [29].

\section{Statistical analysis}

For statistical analysis, IBM SPSS Statistics version 22.0 for Windows software (IBM, Armonk, NY) was used. We assessed differences in patient characteristics using the chi-square test, Student's $t$ test, or analysis of variance when appropriate. Because CSF A $\beta_{42}$, tau, and p-tau were not normally distributed, we logarithmically transformed them for these analyses. Because $A \beta_{42}$ samples were collected over a long period, we rescaled values of $A \beta_{42}$ according to recent insights from our group that $A \beta_{42}$ levels show a significant drift over time [35]. Because of their closely related biological functions, most proteins of the PRM assay were expected to correlate with each other within individuals. Therefore, we used generalized estimating equations (GEEs) with an exchangeable correlation structure, and we clustered the peptides in two levels: all peptides within one subject and all peptides within one protein (i.e., within-subject variables). To achieve a similar order of magnitude in peptide levels, we transformed them to Z-scores for this analysis. Within this model, we analyzed differences of the proteins (entered as dependent variables) between diagnostic groups (entered as independent variables) using a multivariate linear model. To assess if there were differential effects of individual proteins, we included the diagnosis $\times$ protein interaction in the model. Analyses were adjusted for age, sex, $A P O E$ \&4 allele status (dichotomized into no $\varepsilon 4$ alleles or at least one $\varepsilon 4$ allele), and PRM run (to account for day-to-day variation). We recoded diagnostic groups to estimate effect sizes (ß) and SEs for different diagnostic groups. Bonferroni corrections were used to adjust $p$ values for multiple comparisons. To assess which proteins differed between diagnostic groups, we subsequently performed the GEE analysis stratified for individual proteins. In this analysis, each protein (i.e., as a cluster of the peptides from that protein) was assessed in a separate model as a dependent variable. In addition, we repeated all GEE analyses including only the subjects with MCI to compare subjects with sMCI with subjects with MCI-AD. Patients progressing to another type of dementia $(n=3)$ were excluded from this analysis. Last, we assessed associations of the peptides with the established CSF AD biomarkers $A \beta_{42}$, tau, and p-tau using Spearman's correlations. Statistical significance was set at $p<0.05$ for main effects and at $p<0.10$ for interactions.

\section{Results \\ Baseline characteristics}

Table 1 shows the demographic and clinical characteristics of the study population according to baseline and follow-up diagnosis. Patient groups were well matched for age and sex. As expected, patients with AD dementia had the lowest MMSE values, and patients with MCI had values between those of patients with AD dementia and control subjects. CSF $A \beta_{42}$ was lower, and total tau and $\mathrm{p}$-tau levels were higher, in patients with AD dementia, whereas levels of patients with $\mathrm{MCI}$ were between those of patients with $A D$ dementia and control subjects. In patients with MCI-AD, biomarker levels were similar to those of patients with $A D$ dementia, and in patients with sMCI, $\mathrm{A} \beta_{42}$ and tau levels differed only slightly from those of control subjects, but all three biomarker levels were evidently different from those of patients with $\mathrm{AD}$ and patients with MCI-AD.

Table 1 Patient characteristics according to diagnosis

\begin{tabular}{|c|c|c|c|c|c|c|}
\hline & Age, years & Sex, F/M (\% female) & MMSE score at baseline & $\mathrm{CSF} A \beta_{42}(\mathrm{pg} / \mathrm{ml})$ & CSF tau (pg/ml) & CSF p-tau (pg/ml) \\
\hline Control subjects, $n=40$ & $64.5 \pm 8.2$ & $19 / 21(48 \%)$ & $28.3 \pm 1.7$ & $1086 \pm 161$ & $228 \pm 64$ & $40 \pm 9$ \\
\hline $\mathrm{MCl}, n=40$ & $64.5 \pm 8.1$ & 19/21 (48\%) & $26.3 \pm 2.3^{*}$ & $839 \pm 297^{*}$ & $481 \pm 256^{*}$ & $69 \pm 33^{*}$ \\
\hline $\mathrm{sMCl}, n=23$ & $63.7 \pm 7.4$ & $11 / 12(48 \%)$ & $26.7 \pm 2.0$ & $941 \pm 306^{\dagger}$ & $338 \pm 182^{\dagger}$ & $51 \pm 21$ \\
\hline $\mathrm{MCl}-\mathrm{AD}^{\mathrm{a}}, n=14$ & $65.6 \pm 9.0$ & $7 / 7(50 \%)$ & $25.9 \pm 2.7^{\dagger}$ & $632 \pm 84^{* \$}$ & $722 \pm 186^{* \$ \$}$ & $99 \pm 28^{*, \$}$ \\
\hline AD dementia, $n=40$ & $64.6 \pm 8.1$ & 19/21 (48\%) & $21.6 \pm 3.9^{*}$ & $640 \pm 91^{*}, \neq, \$$ & $740 \pm 433^{*}, \neq, \$$ & $94 \pm 47^{*}, \neq, \$$ \\
\hline
\end{tabular}

Abbreviations: $A \beta_{42}$ Amyloid- $\beta$ 1-42, AD Alzheimer's disease, CSF Cerebrospinal fluid, MCI Mild cognitive impairment, MMSE Mini Mental State Examination, p-tau Tau phosphorylated at threonine 181, sMCI Stable mild cognitive impairment

Data are displayed as mean \pm SD unless otherwise indicated. Analysis of variance with post hoc Bonferroni corrections or chi-square tests were used when appropriate. CSF A $\beta_{42}$, total tau, and p-tau were logarithmically transformed for the analyses because of skewed values; in the table, crude values are shown ${ }^{\mathrm{a}} \mathrm{MCl}$ patients progressing to another form of dementia $(n=3)$ were excluded

${ }^{*} p<0.01$ vs controls

${ }^{+} p<0.05$ vs controls

${ }^{+} p<0.01$ vs all $\mathrm{MCl}$ patients

${ }^{\$} p<0.01$ vs sMCl patients 


\section{Differences of proteins between diagnostic groups}

GEE analysis showed a main effect for diagnosis (Wald chi-square $=11.7, p=0.003$ ), as well as an interaction between diagnosis and protein $(p<0.001)$, indicating that the effect for diagnosis differed per protein. Analyses stratified according to protein showed main effects for diagnosis in all proteins but CysC (Fig. 1). Most proteins were elevated in the MCI group, and effect sizes were largest for CHGA ( $\beta=0.63$ [SE 0.17] vs control subjects and $0.54[0.23]$ vs AD), SCG-2 $(\beta=0.53$ [SE 0.20] vs control subjects and 0.74 [SE 0.22] vs AD), NRXN-3 ( $\beta$ $=0.60[\mathrm{SE} 0.20]$ vs control subjects and $\beta=0.65[\mathrm{SE}$ 0.22 ] vs AD), and NPTX1 ( $\beta=0.63$ [SE 0.22] vs control subjects and $\beta=0.78$ [SE 0.21] vs AD). VGF showed a different pattern with evidently lower values in patients with $\mathrm{AD}$ dementia than in control subjects $(\beta=-0.46$ [SE 0.19]) and in patients with MCI (-0.93 [SE 0.22]), whereas the difference between patients with $\mathrm{MCI}$ and control subjects was only borderline significant $(\beta=0.47$ [SE 0.20], $p=0.05$ ). In the GEE analysis comparing patients with sMCI with patients with MCI-AD, there was a main effect for diagnosis as well (Wald chi-square $=6.55$, $p=0.02$ ). The overall interaction between diagnosis and protein was just above the level of significance $(p=0.12)$; however, to be able to compare this analysis with the one with baseline diagnosis, we also stratified this analysis according to protein. This showed main effects for most proteins, except for CysC, LysC, NRXN-2, and NPTX1. The other proteins were elevated in the subjects with MCI-AD compared with the subjects with sMCI, with the largest effect sizes being for CHGA $(\beta=0.98$ [SE 0.26]), NRXN-3 $(\beta=0.96$ [SE 0.35]), and NFASC $(\beta=0.92$ [SE 0.35]). Results of this analysis are shown in Fig. 2.

\section{Correlation with CSF AD biomarkers}

Correlations using Spearman's rank correlation showed that most peptides, except those from LysC, correlated with CSF tau and p-tau in each diagnostic group (see Additional file 2). Generally, in the MCI-AD and AD groups, the correlations with p-tau were stronger than those with tau, whereas in control subjects and subjects with sMCI, tau and p-tau correlated similarly with most peptides. Correlations were stronger between (p)tau and the NRXNs, NPTX1, NFASC, and NCANP than those between (p)tau and the other peptides. Within control subjects and patients with sMCI, most peptides correlated positively with $A \beta_{42}$, whereas they tended to be negative in patients with MCI-AD. Among patients with $A D$, there were no correlations with $A \beta_{42}$.

\section{Discussion}

In this study, we aimed to validate a PRM-MS assay consisting of a panel of proteins presumed to be involved in processes regarding secretory vesicle functioning, synaptic functioning, and innate immunity [12-21]. Having performed a pilot study [29], in the present study, we investigated this panel in a larger and independent cohort that included subjects with MCI in addition to patients with AD dementia and control subjects.

Contrary to our expectations, levels of most proteins were higher in patients with MCI than in the other groups, a finding attributable mainly to those patients who at follow-up had progressed to AD dementia. Stratified analyses showed that these differences were driven mainly by proteins involved in secretory vesicle function (SCG2, CHGA) or synapse formation and stabilization (NRXNs, NPTX1, NCANP), whereas CysC (a cysteine protease inhibitor) was similar between groups and LysC

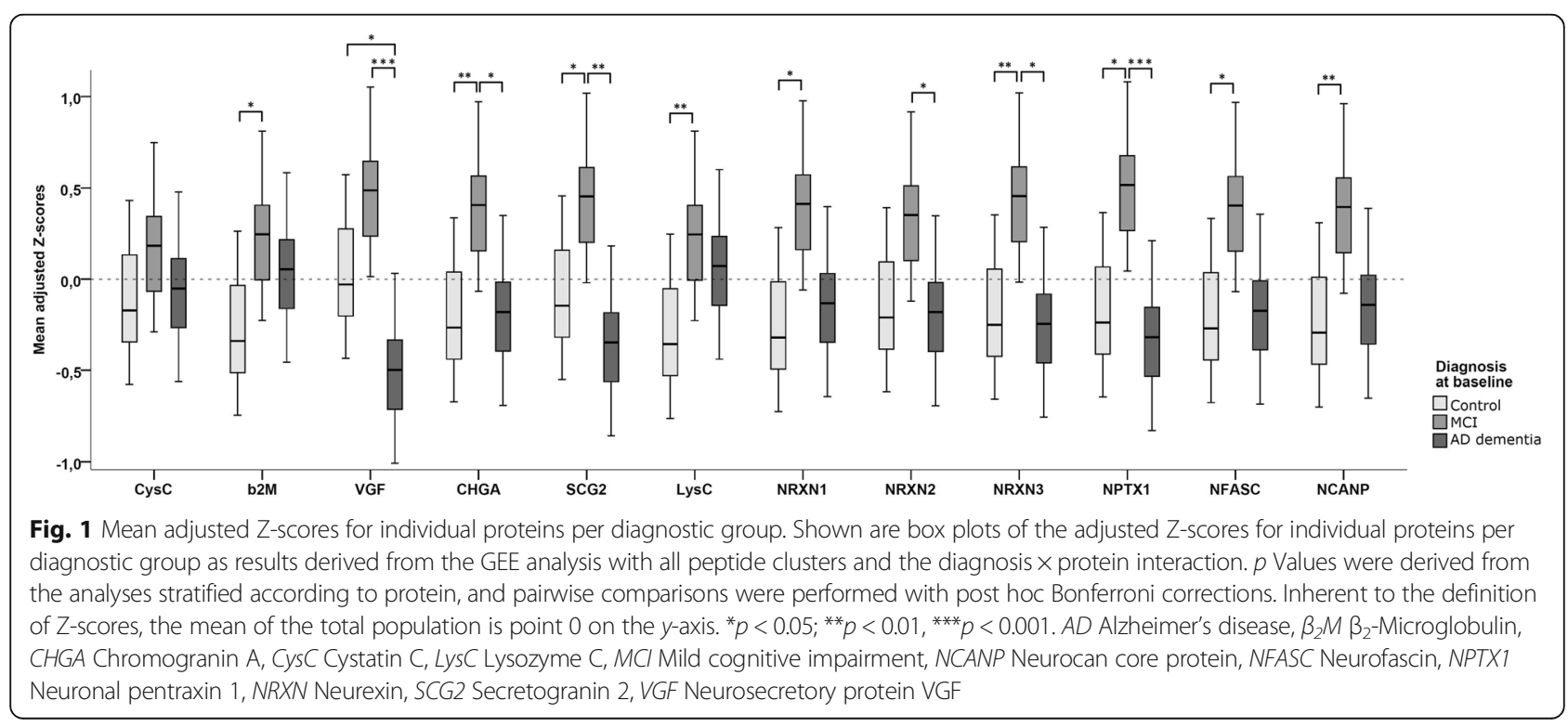




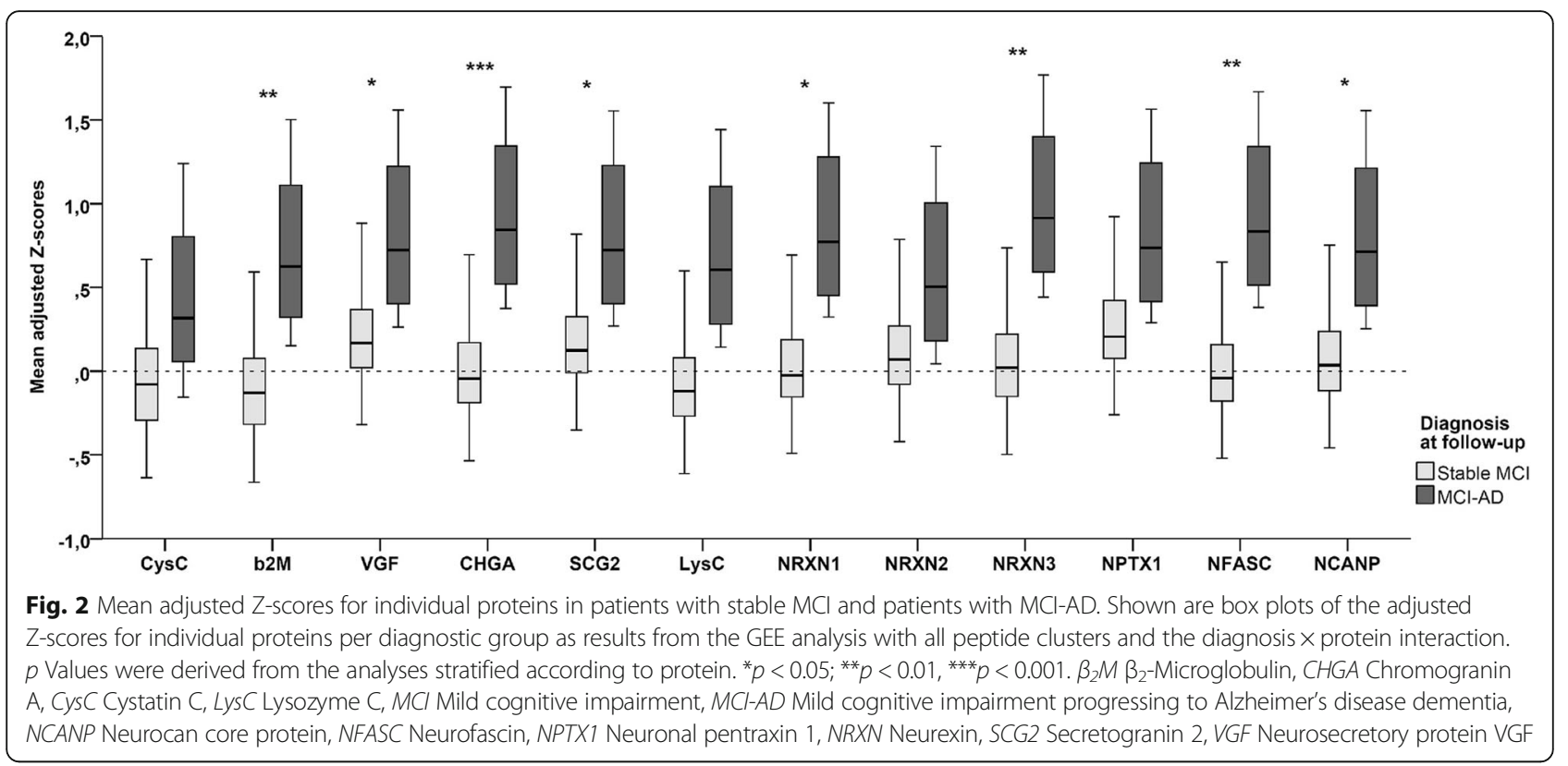

and $\beta_{2} \mathrm{M}$ (both involved in the immune system) showed only small, borderline significant differences. In addition, we found that correlations of most PRM peptides with $A \beta_{42}$ differed markedly between groups, with positive correlations in control subjects and subjects with sMCI but negative correlations in subjects with MCI-AD. In contrast to the findings of our pilot study [29], there were no differences between patients with $\mathrm{AD}$ dementia and control subjects, except for VGF. Importantly, however, the proteins showing differences between groups in the present study were the same proteins as in the pilot study. Hence, the difference between the two studies may be due to differences in the selection of patients or control subjects.

The results regarding VGF in the present study are in line with our pilot study, with clearly lower values in $\mathrm{AD}$ dementia than in the other groups. In the biomarker panel in the present study, VGF has been studied most extensively in $\mathrm{AD}$ research. Lower levels in $\mathrm{AD}$ dementia than in control subjects have been described before [13, 24, 28, 36]. In experiments with hippocampal neurons from rats, VGF peptides increased synaptic plasticity and enhanced proliferation of cells $[37,38]$. Moreover, in another study, VGFknockout mice showed memory impairment [39]. Our findings of lower levels in patients with $\mathrm{AD}$ dementia are hence concordant with these previous studies.

To our knowledge, increased levels of synaptic proteins in patients with MCI due to AD have not been described before. However, on one hand, a recent study showed that higher levels of CHGA in CSF predict future decreases in $A \beta_{42}$ in healthy elderly subjects [25]. On the other hand, a decreased level in brain tissue and CSF of patients with AD compared with control subjects has also been described [27, 28, 40]. Studies on the role of NRXNs, NPTX1, NFASC, and NCANP in AD are rare, although the proteins have all been suggested to have a role in synapse formation, plasticity, and stability [17-21]. On the basis of using MS-based proteomics, CSF NRXN-1 has been found to be slightly decreased in patients with $\mathrm{AD}$ compared with control subjects in a small cohort of 16 subjects [36]. A recent study in mice suggested that NRXN-2 interacts with $A \beta$ oligomers, resulting in loss of synapses, whereas blocking of this interaction prevented $\mathrm{A} \beta$-induced memory impairment [41]. NPTX1 has been found to increase in cell cultures after treatment with $A \beta$, to be present in dystrophic neurites and around $A \beta$ plaques in $\mathrm{AD}$ brains, and to modulate synaptic transmission [42, 43]. A recent study showed that NCANP expression in astrocytes was increased after incubation with $A \beta$ [44]. It has been established that synaptic damage and loss are early events in $\mathrm{AD}$ that are already evident in the MCI stage [5]. Moreover, studies in the early 1990s showed that although the number of synapses declined in early stages of AD, this was first compensated by an increase in synapse size of the remaining synapses and only in later stages was overcome by further synapse loss $[45,46]$. Our finding that the correlations between PRM peptides and $A \beta_{42}$ differed between disease stages also suggests a dynamic pathophysiological trajectory with progression of the disease. The positive correlations in control subjects and subjects with stable MCI suggest an association with normal aging, whereas this association is reversed in cases of $\mathrm{AD}$ pathology. It can be hypothesized that a compensatory mechanism in patients in an early stage of $\mathrm{AD}$ pathology results in upregulation of proteins involved in synaptic plasticity and stabilization, whereas 
concentrations decline in later stages, associated with further synapse loss. Studies finding increased connectivity using functional MRI in amyloid-positive elderly subjects may also point in this direction [47, 48]. Because our AD dementia group consisted of patients still in a relatively early stage of the disease, concentrations at the time of measurement may have been between this elevated level and a subsequent decrease. This was a crosssectional study, however, and longitudinal studies with extensive clinical follow-up and repeated lumbar punctures are needed to assess changes of these proteins within individuals over the course of the disease. Subjects in the earliest disease stages (SCD and MCI at baseline) will be most interesting to investigate for further validation of the present study.

Among the strengths of our study are the relatively large number of patients for this type of experiment and the fact that the cohort was matched for sex and age. The small size of the group with MCI due to AD was a limitation, however, and the results have to be replicated in independent cohorts to be able to draw definitive conclusions. The use of patients with subjective cognitive decline as control subjects in our study is a possible limitation. The advantage, however, is that these subjects received the same cognitive screening as the other patients, ruling out other diagnoses with as much certainty as possible. In addition, the aim was to find CSF biomarkers useful for routine clinical practice; therefore, using perfectly healthy control subjects instead of subjects with SCD might overestimate differences. Strengths of the PRM technique are that it allows for investigation of proteins for which there are no antibodies available and that the specificity is very high. This is important in the search for new biomarkers and for verification of shotgun proteomic findings. A disadvantage, however, is the time needed for analysis, which makes high throughput of a large volume of samples, as would be needed in daily practice, unfeasible.

\section{Conclusions}

Our results suggest that several proteins involved in vesicular transport and synaptic stability are elevated in patients with $\mathrm{MCI}$, especially in patients with MCI progressing to $\mathrm{AD}$ dementia. This may reflect early events in the AD pathophysiological cascade. There were no differences between control subjects and subjects with $\mathrm{AD}$, suggesting that the proteins are probably not suitable as diagnostic biomarkers. However, these proteins may be useful as disease stage or prognostic markers in an early symptomatic stage of the disease, in addition to the core diagnostic markers $A \beta_{42}$ and tau. Whether our findings are consistent, as well as whether these proteins could indeed be used for prognosis, monitoring of disease progression, or assessment of effect of therapy, is a subject for further study.

\section{Additional files}

Additional file 1: Figure S1. Levels of bovine serum albumin per diagnostic group and PRM run. Shown are dot plots of bovine serum albumin added as a reference peptide to monitor sample-processing variations. (PDF $201 \mathrm{~kb}$ )

Additional file 2: Table S1. Correlations of PRM peptides with CSF AD biomarkers. Shown are results of Spearman's rank correlations between PRM peptides and CSF AD biomarkers in each diagnostic group. (DOCX $22 \mathrm{~kb}$ )

\section{Abbreviations}

AD: Alzheimer's disease; APOE: Apolipoprotein $E ; A \beta$ : Amyloid- $\beta$; $A \beta_{42}$ : Amyloid- $\beta$ 1-42; BSA: Bovine serum albumin; $C H G A$ : Chromogranin $A$; CSF: Cerebrospinal fluid; CV: Coefficient of variation; CysC: Cystatin C; GEE: Generalized estimating equation; LysC: Lysozyme C; MCl: Mild cognitive impairment; MCl-AD: Mild cognitive impairment progressing to Alzheimer's disease dementia; MMSE: Mini Mental State Examination; MRI: Magnetic resonance imaging; NCANP: Neurocan core protein; NFASC: Neurofascin; NPTX1: Neuronal pentraxin 1; NRXN: Neurexin; PRM: Parallel reaction monitoring; p-tau: Tau phosphorylated at threonine 181; SCD: Subjective cognitive decline; SCG2: Secretogranin 2; SMCI: Stable mild cognitive impairment; VGF: Neurosecretory protein VGF; $\beta_{2} M$ : $\beta_{2}$-Microglobulin

\section{Acknowledgements}

This study was supported by a grant From Alzheimer Nederland (WE.15-2013-08), the Swedish Research Council, Åhlén-stiftelsen, the European Research Council, Swedish State Support for Clinical Research (ALFGBG), the Swedish Brain Foundation, and the Swedish Alzheimer Foundation, and it is part of the BIOMARKAPD Project within the framework of the European Joint Programme - Neurodegenerative Disease Research (JPND), the Knut and Alice Wallenberg Foundation, the Torsten Söderberg Foundation, Frimurarestiftelsen, Stiftelsen för Gamla Tjänarinnor, Gun and Bertil Stohnes Stiftelse, and Magnus Bergvalls Stiftelse. Research done at the VUmc Alzheimer Center is part of the neurodegeneration research program of Amsterdam Neuroscience. The VUmc Alzheimer Center Amsterdam is supported by Alzheimer Nederland and Stichting VUmc fonds. The clinical database structure was developed with funding from Stichting Dioraphte.

Availability of data and materials

All data used and/or analyzed during the present study are available from the corresponding author on reasonable request.

\section{Authors' contributions}

FHD was responsible for the study design, analysis and interpretation of data, and the writing of the manuscript. GB was responsible for the study concept and design, acquisition of data, and the writing of the manuscript. CET was responsible for critical revision of the manuscript for important intellectual content. $A B$ was responsible for the study design and acquisition of data. PS was responsible for critical revision of the manuscript for important intellectual content. WMVdF was responsible for review of the statistical analysis and critical revision of the manuscript for important intellectual content. $\mathrm{HZ}$ was responsible for the study concept and critical revision of the manuscript for important intellectual content. KB was responsible for the study concept and design, study supervision, and critical revision of the manuscript for important intellectual content. All authors read and approved the final manuscript.

\section{Ethics approval and consent to participate}

All subjects in this study gave written informed consent for the use of their clinical data and CSF for research purposes. The study was approved by the local ethical review board.

Consent for publication Not applicable.

\section{Competing interests}

None of the sponsors had any role in the design and conduct of the study; the collection, management, analysis, and interpretation of the data; the preparation, review, and approval of the manuscript; or in the decision to submit the manuscript for publication. CET is a member of international 
advisory boards of Fujirebio and Roche and has performed contract research for Probiodrug, IBL, and Shire. PS serves or has served on the advisory boards of Novartis, Pfizer, Roche, Danone, Janssen Alzheimer Immunotherapy, Baxter, and Lundbeck. PS also has been a speaker at symposia organized by Lundbeck, Eli Lilly and Company, Merz Pharma, Pfizer, Janssen Alzheimer Immunotherapy, Danone, and Roche. PS is co-editor-in-chief of Alzheimer's Research \& Therapy. PS also is a member of the scientific advisory board of the EU Joint Programme on Neurodegenerative Disease Research (JPND) and the French National Plan Alzheimer. In addition, PS acts as vice-chair of the Dutch Deltaplan for Dementia. PS receives no personal compensation for the activities mentioned above. The other authors declare that they have no competing interests.

\section{Publisher's Note}

Springer Nature remains neutral with regard to jurisdictional claims in published maps and institutional affiliations.

\section{Author details}

${ }^{1}$ Alzheimer Center and Department of Neurology, Amsterdam Neuroscience, VU University Medical Center, P.O. Box 7057, 1007MB Amsterdam, The Netherlands. ${ }^{2}$ Institute of Neuroscience and Physiology, Department of Psychiatry and Neurochemistry, Sahlgrenska Academy at the University of Gothenburg, Mölndal, Sweden. ${ }^{3}$ Clinical Neurochemistry Laboratory, Sahlgrenska University Hospital, Mölndal, Sweden. ${ }^{4}$ Neurochemistry Laboratory and Biobank, Department of Clinical Chemistry, Amsterdam Neuroscience, VU University Medical Center, Amsterdam, The Netherlands. ${ }^{5}$ Department of Epidemiology and Biostatistics, VU University Medical Center, Amsterdam, The Netherlands. ${ }^{6}$ Department of Molecular Neuroscience, UCL Institute of Neurology, Queen Square, London, UK. ' UK Dementia Research Institute at UCL, University College London, London, UK.

\section{Received: 9 November 2017 Accepted: 20 December 2017}

\section{Published online: 15 January 2018}

\section{References}

1. Blennow K, de Leon MJ, Zetterberg H. Alzheimer's disease. Lancet. 2006;368: 387-403.

2. Olsson B, Lautner R, Andreasson U, Ohrfelt A, Portelius E, Bjerke M, et al. CSF and blood biomarkers for the diagnosis of Alzheimer's disease: a systematic review and meta-analysis. Lancet Neurol. 2016;15:673-84.

3. McKhann GM, Knopman DS, Chertkow H, Hyman BT, Jack Jr CR, Kawas CH, et al. The diagnosis of dementia due to Alzheimer's disease: recommendations from the National Institute on Aging-Alzheimer's Association workgroups on diagnostic guidelines for Alzheimer's disease. Alzheimers Dement. 2011;7:263-9.

4. Dubois B, Feldman HH, Jacova C, Hampel H, Molinuevo JL, Blennow K, et al. Advancing research diagnostic criteria for Alzheimer's disease: the IWG-2 criteria. Lancet Neurol. 2014;13:614-29.

5. Scheff SW, Price DA, Schmitt FA, DeKosky ST, Mufson EJ. Synaptic alterations in CA1 in mild Alzheimer disease and mild cognitive impairment. Neurology. 2007:68:1501-8.

6. Janelidze S, Hertze J, Zetterberg H, Landqvist Waldo M, Santillo A, Blennow $\mathrm{K}$, et al. Cerebrospinal fluid neurogranin and YKL-40 as biomarkers of Alzheimer's disease. Ann Clin Transl Neurol. 2016;3:12-20.

7. Calsolaro V, Edison P. Neuroinflammation in Alzheimer's disease: current evidence and future directions. Alzheimers Dement. 2016;12:719-32.

8. Kroksveen AC, Opsahl JA, Aye TT, Ulvik RJ, Berven FS. Proteomics of human cerebrospinal fluid: discovery and verification of biomarker candidates in neurodegenerative diseases using quantitative proteomics. J Proteomics. 2011;74:371-88

9. Brinkmalm A, Portelius E, Ohrfelt A, Brinkmalm G, Andreasson U, Gobom J, et al. Explorative and targeted neuroproteomics in Alzheimer's disease. Biochim Biophys Acta. 1854;2015:769-78.

10. Zetterberg H, Rüetschi U, Portelius E, Brinkmalm G, Andreasson U, Blennow $\mathrm{K}$, et al. Clinical proteomics in neurodegenerative disorders. Acta Neurol Scand. 2008;118:1-11.

11. Maiolica A, Junger MA, Ezkurdia I, Aebersold R. Targeted proteome investigation via selected reaction monitoring mass spectrometry. J Proteomics. 2012;75:3495-513.

12. Bartolomucci A, Possenti R, Mahata SK, Fischer-Colbrie R, Loh YP, Salton SR. The extended granin family: structure, function, and biomedical implications. Endocr Rev. 2011;32:755-97.
13. Carrette O, Demalte I, Scherl A, Yalkinoglu O, Corthals G, Burkhard P, et al. A panel of cerebrospinal fluid potential biomarkers for the diagnosis of Alzheimer's disease. Proteomics. 2003;3:1486-94.

14. Sun B, Zhou Y, Halabisky B, Lo I, Cho SH, Mueller-Steiner S, et al. Cystatin Ccathepsin $B$ axis regulates amyloid beta levels and associated neuronal deficits in an animal model of Alzheimer's disease. Neuron. 2008:60:247-57.

15. Oliveira AL, Thams S, Lidman O, Piehl F, Hokfelt T, Karre K, et al. A role for MHC class I molecules in synaptic plasticity and regeneration of neurons after axotomy. Proc Natl Acad Sci U S A. 2004;101:17843-8.

16. Callewaert L, Michiels CW. Lysozymes in the animal kingdom. J Biosci. 2010; 35:127-60.

17. Dean C, Dresbach T. Neuroligins and neurexins: linking cell adhesion, synapse formation and cognitive function. Trends Neurosci. 2006;29:21-9.

18. Xu D, Hopf C, Reddy R, Cho RW, Guo L, Lanahan A, et al. Narp and NP1 form heterocomplexes that function in developmental and activitydependent synaptic plasticity. Neuron. 2003;39:513-28.

19. Zonta B, Desmazieres A, Rinaldi A, Tait S, Sherman DL, Nolan MF, et al. A critical role for Neurofascin in regulating action potential initiation through maintenance of the axon initial segment. Neuron. 2011;69:945-56.

20. Kriebel M, Wuchter J, Trinks S, Volkmer H. Neurofascin: a switch between neuronal plasticity and stability. Int I Biochem Cell Biol. 2012;44:694-7.

21. Zhou XH, Brakebusch C, Matthies H, Oohashi T, Hirsch E, Moser M, et al. Neurocan is dispensable for brain development. Mol Cell Biol. 2001;21:5970-8.

22. Fagan AM, Perrin RJ. Upcoming candidate cerebrospinal fluid biomarkers of Alzheimer's disease. Biomark Med. 2012;6:455-76.

23. Hansson SF, Andreasson U, Wall M, Skoog I, Andreasen N, Wallin A, et al. Reduced levels of amyloid- $\beta$-binding proteins in cerebrospinal fluid from Alzheimer's disease patients. J Alzheimers Dis. 2009;16:389-97.

24. Jahn H, Wittke S, Zurbig P, Raedler TJ, Arlt S, Kellmann M, et al. Peptide fingerprinting of Alzheimer's disease in cerebrospinal fluid: identification and prospective evaluation of new synaptic biomarkers. PLoS One. 2011;6:e26540.

25. Mattsson N, Insel P, Nosheny R, Zetterberg H, Trojanowski JQ, Shaw LM, et al. CSF protein biomarkers predicting longitudinal reduction of CSF $\beta$-amyloid42 in cognitively healthy elders. Transl Psychiatry. 2013;3:e293.

26. Marksteiner J, Kaufmann WA, Gurka P, Humpel C. Synaptic proteins in Alzheimer's disease. J Mol Neurosci. 2002;18:53-63.

27. Perrin RJ, Craig-Schapiro R, Malone JP, Shah AR, Gilmore P, Davis AE, et al. Identification and validation of novel cerebrospinal fluid biomarkers for staging early Alzheimer's disease. PLoS One. 2011;6:e16032.

28. Simonsen AH, McGuire J, Podust VN, Davies H, Minthon L, Skoog I, et al. Identification of a novel panel of cerebrospinal fluid biomarkers for Alzheimer's disease. Neurobiol Aging. 2008;29:961-8.

29. Brinkmalm G, Sjödin S, Simonsen AH, Hasselbalch SG, Zetterberg H, Brinkmalm A, et al. A parallel reaction monitoring mass spectrometric method for analysis of potential CSF biomarkers for Alzheimer's disease. Proteomics Clin Appl. 2017. doi: 10.1002/prca.201700131

30. van der Flier WM, Pijnenburg YA, Prins N, Lemstra AW, Bouwman FH, Teunissen CE, et al. Optimizing patient care and research: the Amsterdam Dementia Cohort. J Alzheimers Dis. 2014;41:313-27.

31. McKhann G, Drachman D, Folstein M, Katzman R, Price D, Stadlan EM. Clinical diagnosis of Alzheimer's disease: report of the NINCDS-ADRDA Work Group under the auspices of Department of Health and Human Services Task Force on Alzheimer's Disease. Neurology. 1984;34:939-44.

32. Petersen RC. Mild cognitive impairment as a diagnostic entity. J Intern Med. 2004;256:183-94.

33. Albert MS, DeKosky ST, Dickson D, Dubois B, Feldman HH, Fox NC, et al. The diagnosis of mild cognitive impairment due to Alzheimer's disease: recommendations from the National Institute on Aging-Alzheimer's Association workgroups on diagnostic guidelines for Alzheimer's disease. Alzheimers Dement. 2011;7:270-9.

34. Jongbloed W, Kester Ml, van der Flier WM, Veerhuis $R$, Scheltens $P$, Blankenstein MA, et al. Discriminatory and predictive capabilities of enzymelinked immunosorbent assay and multiplex platforms in a longitudinal Alzheimer's disease study. Alzheimers Dement. 2013;9:276-83.

35. Tijms BM, Willemse EAJ, Zwan MD, Mulder SD, Visser PJ, van Berckel BNM, et al. Unbiased approach to counteract upward drift in cerebrospinal fluid amyloid- $\beta$ 1-42 analysis results. Clin Chem. 2017. https://doi.org/10.1373/ clinchem.2017.281055

36. Hölttä M, Minthon L, Hansson O, Holmén-Larsson J, Pike I, Ward M, et al. An integrated workflow for multiplex CSF proteomics and 
peptidomics-identification of candidate cerebrospinal fluid biomarkers of Alzheimer's disease. J Proteome Res. 2015;14:654-63.

37. Thakker-Varia S, Krol JJ, Nettleton J, Bilimoria PM, Bangasser DA, Shors TJ, et al. The neuropeptide VGF produces antidepressant-like behavioral effects and enhances proliferation in the hippocampus. J Neurosci. 2007;27:12156-67.

38. Alder J, Thakker-Varia S, Bangasser DA, Kuroiwa M, Plummer MR, Shors TJ, et al. Brain-derived neurotrophic factor-induced gene expression reveals novel actions of VGF in hippocampal synaptic plasticity. J Neurosci. 2003;23:10800-8.

39. Bozdagi O, Rich E, Tronel S, Sadahiro M, Patterson K, Shapiro ML, et al. The neurotrophin-inducible gene $\mathrm{Vg} f$ regulates hippocampal function and behavior through a brain-derived neurotrophic factor-dependent mechanism. J Neurosci. 2008;28:9857-69.

40. Willis M, Leitner I, Jellinger KA, Marksteiner J. Chromogranin peptides in brain diseases. J Neural Transm. 2011;118:727-35.

41. Brito-Moreira J, Lourenco MV Oliveira MM, Ribeiro FC, Ledo JH, Diniz LP, et al. Interaction of amyloid- $\beta$ (A $A$ ) oligomers with neurexin $2 \alpha$ and neuroligin 1 mediates synapse damage and memory loss in mice. J Biol Chem. 2017;292:7327-37.

42. Abad MA, Enguita M, DeGregorio-Rocasolano N, Ferrer I, Trullas R. Neuronal pentraxin 1 contributes to the neuronal damage evoked by amyloid- $\beta$ and is overexpressed in dystrophic neurites in Alzheimer's brain. J Neurosci. 2006:26:12735-47.

43. Cummings DM, Benway TA, Ho H, Tedoldi A, Fernandes Freitas MM, Shahab $L$, et al. Neuronal and peripheral pentraxins modify glutamate release and may interact in blood-brain barrier failure. Cereb Cortex. 2017;27:3437-48.

44. Yan H, Zhu X, Xie J, Zhao Y, Liu X. B-Amyloid increases neurocan expression through regulating Sox 9 in astrocytes: a potential relationship between Sox9 and chondroitin sulfate proteoglycans in Alzheimer's disease. Brain Res. 2016;1646:377-83.

45. DeKosky ST, Scheff SW. Synapse loss in frontal cortex biopsies in Alzheimer's disease: correlation with cognitive severity. Ann Neurol. 1990;27:457-64.

46. Scheff SW, DeKosky ST, Price DA. Quantitative assessment of cortical synaptic density in Alzheimer's disease. Neurobiol Aging. 1990;11:29-37.

47. Schultz AP, Chhatwal JP, Hedden T, Mormino EC, Hanseeuw BJ, Sepulcre J, et al. Phases of hyperconnectivity and hypoconnectivity in the default mode and salience networks track with amyloid and tau in clinically normal individuals. J Neurosci. 2017;37:4323-31.

48. Jack Jr CR, Wiste HJ, Weigand SD, Knopman DS, Lowe V, Vemuri P, et al. Amyloid-first and neurodegeneration-first profiles characterize incident amyloid PET positivity. Neurology. 2013;81:1732-40.

\section{Submit your next manuscript to BioMed Central and we will help you at every step:}

- We accept pre-submission inquiries

- Our selector tool helps you to find the most relevant journal

- We provide round the clock customer support

- Convenient online submission

- Thorough peer review

- Inclusion in PubMed and all major indexing services

- Maximum visibility for your research

Submit your manuscript at www.biomedcentral.com/submit 